\title{
ZUR STELLUNG DER MODERNEN GEOGRAPHIE
}

\author{
HaNS H. BOESCH
}

Bernhardus Varenius (1622-1650), dessen «Geographica Generalis» 1650 in erster Auflage erschien und 1672 von keinem Geringeren als IsaAc Newton neu herausgegeben wurde, umschrieb im ersten Kapitel «De Geographiae Definitione, Divisione, Methodo et aliis Prolegomenis» in prägnanter Kürze das Objekt der Geographie folgendermaßen: «Objectum geographiae... est tellus, inprimis superficies ejus et partes». Während Jahrhunderten blieb als vornehmste Aufgabe der Geographen die Erforschung unbekannter Gebiete. In dem Maße, wie sich aber die noch weiß verbliebenen Lücken auf unseren Erdkarten schlossen, wandte sich der Erdkundler der eingehenderen Untersuchung der in ihren topographischen Zügen bereits bekannten Teile der Erdoberfläche zu. Wohl gibt es auch heute noch sogenannte weiße Flecken und weite Gebiete, die der eigentlichen Entdeckung harren. So wies vor wenigen Jahren die «New York Times» in einer vielbeachteten Schlagzeile darauf hin, daß wir selbst heutigentags über genauere Karten von der Hälfte des Mondes als von der Hälfte der Erde verfügen; dabei wurde auf die noch sehr ungenügenden Kenntnisse angespielt, welche wir von den vom Wasser bedeckten Teilen unserer Erde oder von rund $70 \%$ der Erdoberfläche besitzen! Auch die in vollem Gange befindliche Erforschung der Antarktis zeigt, daß die Periode der eigentlichen geographischen Entdekkungen keineswegs als abgeschlossen betrachtet werden kann. Das ändert jedoch nichts daran, daß für die meisten Geographen das Forschungsziel heute in erster Linie in der vertieften Erkenntnis und im besseren Verstehen des in den großen Zügen schon Bekannten liegt. Wenn auch das Objekt der Untersuchung, wie es in klassischer Klarheit von VARENIUS definiert wurde, im Wesentlichen das gleiche geblieben ist, so haben sich doch die Methoden und im Einzelnen die Ziele der wissenschaftlichen Untersuchung in neuerer Zeit ganz bedeutend gewandelt. Allein schon aus diesem Grunde nehmen in der modernen geographischen Literatur grundsätzliche Arbeiten, welche sich mit den angegebenen Hauptproblemen des Objektes, der Einteilung und den Untersuchungsmethoden befassen, einen breiteren Raum ein als in anderen Wissensgebieten. Dabei spielen freilich auch noch andere Gründe, auf welche im Folgenden eingetreten wird, entscheidend mit.

Wenn VARENIUS von der «superficies» sprach, dann meinte er damit freilich ebensowenig wie der moderne Geograph die nackte, von allem Beiwerk des Menschen, von aller Vegetation und allem tierischen Leben entblößte Erdoberfläche. In dieser reduzierten Form ist sie vielmehr das Objekt einer eigenen Wissenschaft, der Geomorphologie, und wird von ihr in gleich unabhängiger Weise untersucht wie die Hydrosphäre, die Atmosphäre usw. im Rahmen ihrer entsprechenden Wissensgebiete. Das Besondere des geographischen Untersuchungsobjekts liegt gerade darin, daß die verschiedensten Sphären sich in ihm zu einem Ganzen vereinigen, welches sowohl in seinen Teilen als auch in deren gegenseitigen Beziehungen zu studieren ist. Das ist es, was VARENIUs unter «superficies» verstand und das in neuerer Zeit der bekannte deutsche Geograph A.HeTTNER mit «Erdhülle» bezeichnet. Seither ist - nicht nur in der deutschen Sprache dafür der Ausdruck «Landschaft» aufgekommen, der aber wegen seiner Vieldeutigkeit wohl besser aus der wissenschaftlichen Literatur wieder verschwinden sollte. Besser schiene uns, in Anlehnung an das oben Gesagte, einen «terminus technicus», wie beispielsweise «Geosphäre», neu zu schaffen.

$\mathrm{Zu}$ Beginn der Neuzeit und bis in die zweite Hälfte des vergangenen Jahrhunderts war es möglich, daß einzelne hervorragende Gelehrte sowohl das Ganze überschauen als auch in den verschiedenen Teilgebieten forschend tätig sein konnten. Im Jahre 1959 wurde der hundertste Todestag Alexander von Humboldts gefeiert, den wir wohl als den bedeutendsten Vertreter dieser Epoche betrachten dürfen. Seine Expedi- 
tionsberichte der großen Südamerikareise legen Zeugnis von einer sämtliche Teilgebiete der Geosphäre erfassenden Forschungstätigkeit ab, wobei, dem Untersuchungsgebiet entsprechend, die naturwissenschaftlichen Untersuchungen im Vordergrund standen. Es sei aber ebenso zu beachten, daß HuмвоцDт immer anstrebte, die Einzelerscheinungen im größeren geographischen Zusammenhang zu sehen. Seine «Ansichten der Natur» und der «Kosmos» legen davon schönstes Zeugnis ab. So ist es durchaus richtig, wenn im Jahre 1959 in erster Linie die Geographen der ganzen Welt dieses hervorragenden Gelehrten gedachten.

Eines der Hauptprobleme nicht nur der Geographie, sondern der Wissenschaft überhaupt liegt in der zunehmenden Spezialisierung, welche durch den immer ausgedehnteren Erkenntnisbereich gefordert wird. Die Geographie empfindet diese Entwicklung mit besonderer Schärfe, da im geosphärischen Bereich mehr und vielseitigere Dinge zusammenkommen als irgendwo sonst. Die Fülle des vorhandenen Tatsachenmaterials bedrängt den Geographen in einem solchen Maße, daß er je länger je mehr gezwungen ist, seine eigentliche Forschungstätigkeit an der Peripherie, das heißt in den einzelnen Teilgebieten, einzuschränken oder gar ganz aufzugeben und sich in erster Linie auf die Besonderheiten, welche durch das Zusammentreten der einzelnen Bausteine entstehen, zu konzentrieren. Zahlreiche Teilgebiete, die früher im Rahmen der Geographie gepflegt wurden, sind zu eigenen Wissenschaften geworden, ein Prozeß, der sich in Zukunft ohne Zweifel fortsetzen wird. Man hat der Geographie deshalb schon den Vorwurf gemacht, daß sie kaum mehr eigene Forschung betreibe, vielmehr alles Tatsachenmaterial aus zweiter Hand beziehe. Dabei übersieht man allzu leicht, daß dieses Ordnen und Zueinander-in-Beziehung-Bringen ebenfalls wissenschaftliche Forschung im besten Sinne ist und ganz bestimmten methodologischen Prinzipien gehorcht. Die heutige Situation kann vielleicht so charakterisiert werden, daß praktisch alle wissenschaftlich tätigen Geographen noch in der Lage sind, sich auf irgendeinem Teilgebiet als Spezialforscher zu betätigen. In erster Linie wären dabei etwa die Geomorphologie, die Klimatologie, die Pflanzengeographie oder Einzelgebiete der Human Sciences zu nennen. Die Zeiten einer wahrhaft umfassenden Beherrschung aller Sphären sind aber auf immer entschwunden.

Ein weiteres Problem der Geographie liegt in ihrem dualistischen Charakter. Natur und Mensch tragen beide zur Gestaltung der Erdoberfläche bei, und beide sind als Faktoren bei der geographischen Untersuchung entsprechend $\mathrm{zu}$ würdigen. Wohl mag, Zeitströmungen folgend, das Pendel bald mehr in der einen, bald mehr in der andern Richtung ausschlagen. Um die Jahrhundertwende spielte die naturwissenschaftliche Richtung in der deutschen Geographie die Hauptrolle und färbte noch auf Jahrzehnte in der Schulgeographie so stark ab, daß vom Laien oft Geographie mit Oberflächengeologie gleichgesetzt wurde. Doch fehlte auch die Gegenströmung nicht, wie in unserer Zeit beispielsweise die Entwicklung der Sozialgeographie in Holland, den Vereinigten Staaten oder anderswo zeigt. Wenn wir versuchen, unvoreingenommen die Verhältnisse zu überprüfen, gelangen wir zu folgenden Schlüssen: Eigentliche Naturlandschaften ohne jede Beeinflußung durch den Menschen, bei deren Studium rein naturwissenschaftliche Methoden angewandt werden, werden immer seltener. Das $\mathrm{Zu}$ sammenspiel der einzelnen Elemente zum geosphärischen Ganzen folgt in diesem Falle ausschließlich naturwissenschaftlicher Kausalität. Am anderen Ende der Skala stehen jene Gebiete, welche auf das intensivste vom Menschen mitgestaltet wurden. Sofern wir dem Menschen die Möglichkeit einer freien Entscheidung zubilligen, müssen wir ihn, um zu einer richtigen Deutung zu gelangen, als Einzelindividuum wie als Glied der menschlichen Gesellschaft in seinem besonderen und von den Naturfaktoren grundsätzlich verschiedenen Einflu $\beta$ würdigen. $Z$ wischen diesen beiden Polen finden wir auf der Erde sämtliche Übergänge. Ohne daß sich dies etwa in exakten Zahlen ausdrücken ließe, kann wohl in erster Annäherung gesagt werden, daß der Geograph in gleichem Maße den «Earth Sciences» wie den «Human Sciences» verpflichtet ist. 
Unsere Hochschulen - und diese Feststellung gilt für die ganze Erde - gliedern dagegen die Wissensgebiete organisatorisch traditionell in Natur- und Geisteswissenschaften, wobei, wie etwa in Zürich wichtige, die Geographie direkt berührende Teilgebiete außerdem den Wirtschaftswissenschaften zugeordnet werden. Es ist im Auslande schon da und dort darauf hingewiesen worden, da $\beta$ die besonderen Voraussetzungen, welche die Geographie mit sich bringt, im Grunde für sie eine eigene Stellung zwischen oder über den Fakultäten, etwa im Rahmen eines Geographischen Institutes eigener Art, verlangen. Die Lösungen, welche an den einzelnen Hochschulen hinsichtlich dieses überall auftretenden Problems getroffen werden, weichen entsprechend den örtlichen Verhältnissen stark voneinander ab. In Zürich ist die Geographie der naturwissenschaftlichen Fakultät (philosophische Fakultät II) eingegliedert, ohne daß sich daraus irgendwelche Schwierigkeiten ergeben hätten, tragen doch in freizügiger Weise die Prüfungsbestimmungen und die verständnisvolle Haltung der verantwortlichen Kreise den besonderen Belangen der Geographie voll Rechnung.

Den Niederschlag dieser Verhältnisse finden wir nicht zuletzt im Ausbau der verschiedenen geographischen Hochschulinstitute. Selten sind heute glücklicherweise die Fälle, in denen die Betreuung des Gesamtgebietes nur einem Professor obliegt; meistens teilen sich mindestens zwei Professoren in Lehre und Forschung. Dabei erfolgt die Aufteilung in der Regel in Natur- und Anthropogeographie. Vom Standpunkte der Einheit der Geographie aus dürfte es dagegen sinngemäßer sein, wenn jeder der Dozenten seinen Teil aus beiden Hauptgebieten übernähme. Mit Bezug auf die materiellen Voraussetzungen sind heute die meisten Institute so ausgerüstet, daß sowohl Laboratorien für die rein naturwissenschaftlichen Belange wie auch die entsprechenden Hilfsmittel für die anthropogeographische Forschung zur Verfügung stehen. Dies ist schon im Interesse der Ausbildung notwendig, die zweckmäßig auf einer soliden naturgeographischen Basis aufbaut.

Damit hätten wir vorerst auf einige Fragen, welche die Stellung der Geographie im Rahmen der übrigen Wissenschaften und der Hochschulen betreffen, hingewiesen. Wir wenden uns im Folgenden einigen Problemen zu, welche mehr das innere Gefüge der Geographie und das geographische Arbeiten selbst betreffen.

Nachdem wir uns bisher schon über das Objekt und das Forschungsziel der Geographie geäußert haben, greifen wir nun aus verschiedenen wissenschaftlichen geographischen Zeitschriften eine größere $Z$ ahl von Arbeiten heraus, um zu untersuchen, in welchem $\mathrm{Maße}$ und in welcher Weise sich die Autoren dem genannten Ziel unterstellten. Wir werden dabei feststellen, daß nur wenige Arbeiten in abgewogener Weise die Geosphäre als Ganzes und ihre Elemente untersuchen. In den meisten Fällen wird es sich um Arbeiten zu Teilfragen handeln oder - im Sinne der Geographie - um Elementaruntersuchungen. Das ist an sich nichts Erstaunliches und dürfte sich wohl in den meisten Wissengebieten ähnlich verhalten. Geographisch bleiben solche Untersuchungen jedoch immer, wenn sie im Hinblick auf das Ganze durchgeführt und ihre Resultate entsprechend gewürdigt werden. Andernfalls wären sie in einer geographischen Zeitschrift am unrichtigen Platz. Gleichgültig, ob ein Geograph integrierte landschaftskundliche Studien betreibt oder sich mit Teiluntersuchungen befaßt, im Hintergrund steht für ihn immer das eingangs umschriebene Forschungsziel.

Die landschaftliche Analyse an sich bietet keine Probleme besonderer Art. Die Landschaft im Sinne des Geographen ist ein derart komplexes Gebilde, daß ihre Untersuchung nur so möglich ist, $\mathrm{da} ß$ sie vorerst in ihre einzelnen Teile zerlegt wird (Elementaranalyse). Bei der folgenden Darlegung der Untersuchungsergebnisse sind lediglich jene Dinge erwähnenswert, welche mit Bezug auf die angestrebte Synthese relevant sind. In dieser feinen Nuancierung zeigt sich ein wesentlicher Unterschied in der Bearbeitung eines solchen Teilgebietes durch einen Geographen oder durch einen Spezialisten. Die Ausbildung eines Geographen und damit auch seine späterc Blickrichtung konzentriert sich darauf, daß das Einzelne - auch wenn es Mittelpunkt 
einer gerade durchgeführten Arbeit ist - immer im größeren Zusammenhang des geosphärischen Ganzen gesehen und gewürdigt werde. Teilweise wird der Geograph in der Lage sein, die entsprechenden Untersuchungen selbst auszuführen; in vielen Fällen wird er aber darauf angewiesen sein, die Untersuchungen anderer, die Spezialisten in diesen Teilgebieten sind, herbeizuziehen und geographisch auszuwerten. Anders steht es mit der Synthese, welche beträchtliche methodische und darstellerische Schwierigkeiten bietet. Logisch erscheint zweifellos jener Weg als richtig, welcher der analytischen Betrachtung der Landschaftselemente eine ebenso detaillierte Betrachtung der zwischen diesen bestehenden Bindungen und Beziehungen folgen läßt.

Gerade an diesem Punkte macht sich der dualistische Charakter der Geographie erneut mit aller Schärfe bemerkbar: Solange wir lediglich Naturlandschaften untersuchen, werden diese Beziehungen sich dem naturwissenschaftlichen Kausalitätsprinzip unterordnen, während in der Kulturlandschaft der menschliche Wille entscheidend mitspielt. Dem Geographen fällt deshalb die schwierige Aufgabe zu, bei der landschaftskundlichen Untersuchung diese beiden Prinzipien im richtigen Verhältnis und an der richtigen Stelle anzuwenden. In der Naturlandschaft wird sich, sofern die mitspielenden Faktoren unverändert bleiben, mit der Zeit ein Endzustand einspielen, der in Anlehnung an die Pflanzengeographie als Klimax-Naturlandschaft bezeichnet werden könnte. In der Kulturlandschaft hängt, wofür wissenschaftlich genügend Belege beigebracht werden könnten, die Bejahung einer solchen Möglichkeit in erster Linie davon ab, welche philosophische Grundhaltung wir einnehmen.

Nach den hier vertretenen Auffassungen dürfte in der Kulturlandschaft kaum ein Analogon zur erwähnten Klimax-Naturlandschaft zu erwarten sein. Dieser Unterscheidung kommt freilich mehr nur akademische und die Spannweite geographischer Arbeit beleuchtende Bedeutung zu, da auch in der Naturlandschaft ein kontinuierlicher Wechsel der bedingenden Faktoren (wie Klima, 'Topographie usw.) zu einem ständiger Veränderung unterworfenen geosphärischen Ganzen führt.

Das geographische Arbeiten besteht demnach im wesentlichen darin, an einzelnen Punkten der Erdoberfläche mit Hilfe von Analyse und Synthese einzelne individuelle Ausbildungen der Superficies, der Erdhülle, Landschaft oder Geosphäre - wie immer wir das Objekt benennen mögen - zu beschreiben und zu verstehen. In Analogie zu anderen Wissenschaften führen diese Untersuchungen schließlich zur Aufsammlung eines äußerst reichhaltigen Materiales, welches anschließend einer systematischen Ordnung unterworfen werden sollte. Dabei wäre, bestimmten wissenschaftlich vertretbaren Prinzipien folgend, das untersuchte Individuum durch die Angabe typischer Werte, welche einer Vielzahl unter ihnen eigen sind, zu typisieren und zu klassifizieren. Hier machen wir die erstaunliche Feststellung, daß es in der wissenschaftlichen Gcographie bislang noch kein auch nur einigermaßen anerkanntes Ordnungsprinzip gibt. Mehrfach waren die Versuche, ähnlich den biologischen Wissenschaften eine Gliederung hierarchischer Art in Familien/Gattungen/Arten durchzuführen; dabei wurde übersehen, daß das dieser Gliederung zugrunde liegende biologisch-genetische Prinzip im vorliegenden Falle nicht angewendet werden kann. Für Naturlandschaften schiene eine Gliederung nach Dominanten und zusätzlichen Elementen wie in der Petrographie vertretbar, doch scheiterten solche Versuche bisher an der großen Zahl der relevanten Komponenten, wie auch daran, daß ein und dasselbe Element bald bedeutungsmäßig hervor-, bald zurücktritt. Nicht zuletzt dürfte das Fehlen einer Systematik und Landschaftstypologie auch darin zu erblicken sein, daß im Falle der Geographie der Schritt vom Individuum zum Typus nur zögernd getan wird, weil nach der Ansicht namhafter Vertreter dabei Wesentliches unter den Tisch fällt. Einer der ersten erfolglosen Versuche zu einer Typisierung stammt von dem Engländer A. J. Herbertson, welcher 1905 in der Royal Geographical Society über «The Major Natural Regions: An Essay in Systematic Geography» vortrug. Ihm, dem geplagten Lehrer, schwebte in erster Linie vor, die Vielfalt des zu behandelnden Materials mit Hilfe der Typisie- 
rung auf ein der verfügbaren Stundenzahl entsprechendes Maß zu reduzieren. Aber auch andern Versuchen, bei denen die Notwendigkeit der wissenschaftlichen Ordnung mehr im Vordergrund stand, war bisher nicht mehr Erfolg beschieden. Unseres Erachtens liegt die Hauptschwierigkeit außer in den bisher behandelten Gründen vor allem darin, daß noch kein vernünftiges und wissenschaftlich brauchbares Grundprinzip - vergleichbar dem biologisch-genetischen - gefunden wurde. Ein solches könnte beispielsweise für eine erste Gliederung in der Zahl der vertretenen Sphären gesehen werden. Versuche von Bobek, Carol u.a.m. weisen in dieser Richtung. Doch handelt es sich dabei erst um vorläufige und ganz generelle Versuche zu einer systematischen Ordnung.

Eine überzeugende Typisierung hätte nicht zuletzt auch den großen Vorteil, daß sie eine Gliederung der Erdoberfläche in Gebiete gleicher typischer Ausstattung erlauben würde. Alle bisherigen Gliederungen dieser Art sind entweder von einem bestimmten Blickpunkt aus vorgenommen worden oder beschränken sich auf eine naturlandschaftliche Gliederung.

Freilich müssen wir an diesem Punkte noch auf eine weitere Besonderheit hinweisen, welche der geographischen Untersuchung eigen ist. In unserer bisherigen Betrachtung stellten wir fest, daß die Untersuchungen der Geosphäre von den Verhältnissen an einem bestimmten Punkt ausgehen und durch Zusammenfassung von Gleichem zur räumlichen Gliederung fortschreiten. Es liegt dabei in der Natur des Untersuchungsobjektes, daß es lediglich von den örtlichen Gegebenheiten, vom Grade der Typisierung und vom Maßstab abhängt, ob die so erhaltene Aufgliederung feiner oder gröber wird. Offensichtlich gibt es im geosphärischen Bereich keine «kleinsten Landschaftszellen», die man nicht mehr weiter unterteilen kann, ohne da $\beta$ sie Wesentliches verlieren (so wie etwa ein Organismus bei weiterer Zergliederung in seine einzelnen Organe zerfällt). Neben die bisher geschilderte Betrachtungsweise stellt sich jedoch eine zweite, die in neuerer Zeit immer größere Beachtung erheischt. Außer den Bedingungen, welche an ein und demselben Punkte zwischen den Landschaftselementen bestehen, haben wir jene Beziehungen zu untersuchen, welche vor allem durch den Menschen zwischen verschiedenen Punkten der Erdoberfläche in vielfältigster Weise geschaffen worden sind. Neben die Gliederung der ersten Art stellt sich damit ein komplexes System von organisatorischen räumlichen Einheiten, die durcheinander, nebeneinander und übereinander über die Erdoberfläche gelegt wurden. Der Naturlandschaft fehlen solche Raumgebilde beinahe völlig, in der Anthropogeographie nehmen sie aber einen wichtigen Platz ein. Produktions- und Konsumationsgebiete, das Hinterland von Häfen, die Einzugsgebiete von Städten gehören ebenso hierher wie das Wirtschaftsareal eines Dorfes und anderes mehr. Es ist ganz zweifellos, daß die Untersuchung dieser Dinge in den Bereich der geographischen Forschung gehört, bilden sie doch einen integrierenden Bestandteil der vom Menschen gestalteten Landschaft. Freilich verlangen sie völlig andere Untersuchungs- und Darstellungsmethoden, und ihre Einbeziehung in das geographische Schaffen vergrößert in jeder Weise die ohnehin schon bestehende Weite der Probleme. Um die beiden Betrachtungsrichtungen klar zu unterscheiden, bezeichnet man die zuletzt besprochene als die funktionale, weil sie funktionale Raumgebilde untersucht und zur Aufdeckung funktionaler Strukturen führt; die zuerst besprochene wird per analogiam als formale (formale Struktur usw.) bezeichnet.

Unabläßig wandelt sich die Landschaft; meist langsam und über Jahrtausende und Jahrmillionen die Naturlandschaft, bedeutend rascher die Kulturlandschaft dagegen. Wenn wir jedoch innerhalb der Kulturlandschaft den Wandel der formalen und der funktionalen Strukturen vergleichen, stellen wir fest, daß letztere oft einen geradezu stürmischen Wechsel und Wandel zeigen. Von einem Tag auf den andern können funktionale Strukturen durch Eingriff des Menschen tiefgreifend umgestaltet werden, während die im Materiellen verankerten formalen Strukturen sich erst langsam 
der neuen Situation anzupassen versuchen. Eines der faszinierendsten Probleme der modernen Anthropogeographie stellt zweifellos dieses Wechselspiel dar. In Zeiten rascher wirtschaftlicher Entwicklung und Ausweitung bemühen wir uns ständig, die übernommene formale Struktur der Landschaft mit der neugeschaffenen funktionalen Struktur wieder in Übereinstimmung zu bringen. Wir nennen dies Modernisierung, Innenkolonisation, Integralmelioration ... oder wenn wir, Zukünftiges vorwegnehmend, weiter blicken, Regional- und Landesplanung.

Gleichzeitig führen aber die Eingriffe in die formale Struktur dazu, daß ein unter Umständen seit langem eingespieltes Gleichgewicht in tragischer Weise gestört wird und sich als Folge einer Kettenreaktion unvorhergesehene Störungen ergeben. Dies ist, wie bekannt, vor allem dort der Fall, wo mit der Ausweitung der Ökumene der Mensch auf die noch bestehende Naturlandschaft übergreift. Der Geograph steht allen diesen Veränderungen in ähnlicher Weise wie der Arzt dem kranken Körper gegenüber; was hier in gärendem Wandel begriffen ist und in irgendeiner Weise wieder in Einklang gebracht werden sollte, war seit jeher sein Untersuchungs- und Forschungsobjekt, das er sowohl in seinen Teilen wie auch als Ganzes zu verstehen versucht.

\section{LA GEOGRAPHIE MODERNE}

La géographie moderne voit aujourd'hui son but principal dans la géosphère qui est composée par les différentes sphères partielles (superficies, paysages, etc.). Ainsi, le centre de gravité des recherches géographiques se déplace de la spécialisation des différentes branches (géomorphologie, etc.) à l'étude des structures géosphériques. Conformément à son double caractère, la géographie travaille, d'un côté, essentiellement avec des méthodes scientifiques naturelles (paysages naturels), tandis qu'à l'étude des paysages humanisés, elle a, de l'autre côté, à respecter la volonté indépendante de l'homme. Quoi que les méthodes pour l'étude des structures géosphériques formelles et fonctionnelles soient déjà fort avancés, il manque, à l'heure actuelle, un principe d'ordre systématique. A cet égard, relevons les essais de Bobek, Carol, etc. d'obtenir

un ordre systématique sur la base des sphères d'un paysage et de l'intensité de leur relation mutuelle.

\section{DIE GEOGRAPHIE \\ AN DER I42. JAHRESVERSAMMLUNG DER SCHWEIZERISCHEN NATURFORSCHENDEN GESELLSCHAFT}

\section{ERNST WiNKLER}

Die 142. Jahresversammlung der SNG fand vom 7.-9. September 1962 im Unterengadin statt. Wie gewohnt beteiligte sich der Verband der Schweiz. Geogr. Gesellschaften unter seinem Präsidenten Prof. Dr. H. Gutersohn an ihr, indem er eine Sektion «Geographie und Kartographie» sowie eine Orientierung über das Tagungsgebiet und eine Exkursion ins Samnaun organisierte.

Der Jahresvorstand, den der Direktor des Hochalpinen Töchterinstituts Ftan, Dr. M. Gschwind, umsichtig. leitete, hatte zum Thema der Hauptvorträge die Frage der Möglichkeiten, Grenzen und Verantwortung in der wissenschaftlichen Forschung gewählt. Zu ihm sprachen der Mediziner Prof. Dr. H. Fischer, Zürich, und der Physiker Prof. Dr. M. Fierz, Zürich. Die wissenschaftliche Sitzung der Geographen wurde am Morgen des 8. Septembers im Schulhaus Scuol abgehalten. Es sprachen 6 Referenten, deren Vorträge anschließend resümiert sind.

In der am gleichen Morgen abgehaltenen Delegiertenversammlung des VSGG orientierte der Zentralpräsident über die angelaufenen Arbeiten zum Landesatlas, dem ein Büro in der ETH unter Leitung von J. RoTH mit den Mitarbeitern B. RüDIN und H. Leutzinger zur Verfügung steht. Die Oberleitung haben Prof. Dr. h.c. E. Imhof und Prof. Dr. H. Gutersohn. Auch der Exkursionsführer soll gefördert werden. Nachdem ein thematisches Beispiel publiziert wor- 